\title{
Steady State Analysis of M/G/1 and M/Er/1 Line Models with MATLAB Environment in Cloud Computing Applications
}

\author{
${ }^{1} \mathrm{~N}$. Thirupathi Rao, ${ }^{1}$ Debnath Bhattacharyya, ${ }^{2}$ Midhunchakkaravarthy and ${ }^{3}$ Tai-Hoon Kim \\ ${ }^{1}$ Department of Computer Science and Engineering, \\ Vignan's Institute of Information Technology (A), Visakhapatnam, Andhra Pradesh (AP), India \\ ${ }^{2}$ Faculty of Computer Science and Multimedia, Lincoln University College, Malaysia \\ ${ }^{3}$ Sungshin Women's University, Bomun-ro 34da-gil, Seongbuk-gu, Seoul, Korea
}

\begin{abstract}
Distributed computing is the way toward designating the system get to admission to a gathering of chosen clients. These clients might having progressed and savvy example of processing works or tasks on the arrangement of convenience of the system consent for getting to the system assets at whatever point. By and large the diagnostic models were intended for thinking of the models that utilization the cloud and its administrations through that the execution of the model. The model was broke down and assessed underneath various arrangements and suppositions. These presumptions depended on the lining hypothesis and its exactness is checked with numerical figures and reenactments. Current paper discusses the execution assessment as far as relentless state parameters of a little cloud server cultivate utilizing single and multi-server lining models. Single server display incorporates $\mathrm{M} / \mathrm{G} / 1$ and $\mathrm{M} / \mathrm{Er} / 1$. Multi-server models considered incorporate $\mathrm{M} / \mathrm{G} / \mathrm{c}, \mathrm{M} / \mathrm{Er} / 1$. An examination among the enduring state parameters assessed for the above lining models as for activity force is likewise exhibited.
\end{abstract}

Key words: Cloud computing, M/G/1 Model, M/Er/1 Model, M/G/c Model and M/G/c/c Models, throughput, bandwidth, MATLAB and traffic strength

\section{INTRODUCTION}

New patterns identified with personnel computers innovation develop consistently and one of those new patterns was distributed computing. An aggregate programming and its related applications and necessary conditions considered under distributed computing are discussed. Some of the major applicants of these models are Amazon, Google application motor and IBM had used. An essential part of distributed computing is Infrastructure-as-a-benefit (IaaS) (Sztrik, 2012) which is the capacity to get to registering assets remotely. The remote access of the administrations given by the distributed computing condition to the system. Average cases of IaaS incorporate PC cycles, servers, stockpiling, system and reinforcement and so on. One of the benefits of IaaS is that one can get to extremely costly server farm assets through a rental means.

The most and the essential point to be considered was the distributed computing can be taken as the imperative angle for both the suppliers of the cloud and the clients of the distributed computing. The unfaltering state execution parameter details distinguished are modified in MATLABß condition (Hlynka and Molinaro, 2010). The present models chose for assessment for single servers incorporate $\mathrm{M} / \mathrm{G} / 1$ and $\mathrm{M} / \mathrm{Er} / 1$. The multi-server models considered were $\mathrm{M} / \mathrm{M} / \mathrm{c}, \mathrm{M} / \mathrm{G} / \mathrm{c}$ and $\mathrm{M} / \mathrm{Er} / 1$. Interarrival rates for all the above models have exponential dispersion and have more extensive scope of conveyances including exponential, summed up, deterministic and Erlang write.

Literature review: An extensive survey on cloud computing highlighted by Mary and Jayapriya (2014) where a comprehensive survey on quality of service concerning their implementation details, sharp points and limitations were presented. William Stallings provided a practical guide to queuing analysis and also reviewed some elementary concepts in probability and statistics. Presentation points of cloud computing information stations using ((M/G/1): (Inf/GD Model)) queuing system were arrived by Mary and Saravanan (2013) mainly for a mean number of tasks and waiting time in the system.

Corresponding Author: N. Thirupathi Rao, Department of Computer Science and Engineering, Vignan's Institute of Information Technology (A), Visakhapatnam, Andhra Pradesh (AP), India 
Queuing models are instrumental in the working and processing of specific models and the data related to several applications like the intend of manufacture, shipping, transport and stocking systems regarding capacities and control. Fundamental concepts in queuing theory documented by Khanghahi and Ravanmehr (2013). Both finite source and infinite source queuing models dealt in the above reference. Mathew (2014) surveyed on a variety of machines and models those used for studying and identifying the data centre and its requirements and the performance, evaluation for providing the quality of service in laaS cloud computing and it is related using systems. Transient probabilities of $\mathrm{M} / \mathrm{M} / 1$ queue calculated by Sowjanya et al. (2011) based on task time many customers and traffic intensity. Brandwajn and Wang (2008) brought out an on the whole standpoint of cloud estimation criteria and tinted it with an assist of simulation.

Mathew (2014) proposed and analysed a set of new queuing patterns which could encounter while working on these models and the classification part of these patterns discussed. Sowjanya et al. (2011) verified and proved that $\mathrm{M} / \mathrm{M} / \mathrm{c}$ Model for two servers which increase the performance of the network model over using one server by tumbling the length of the queue and waiting time. Tripathi (2013) measured an M/G/1 -like system in which the service time distribution presented by a coxian series of various memory fewer stages. A new and novel approach based on conditional probabilities is used to obtain a solution of such systems.

An exhibition of academic outcome for the $\mathrm{M} / \mathrm{M} / \mathrm{c} / \mathrm{c}+\mathrm{r}$ queuing model with eager clients is offered by Hideaki Takagi et al. with mathematical design as a fundamental form of call centres including derivations of combined sharing for the waiting time and probability of customer service and blocking. Ward Whitt (Mary and Jayapriya, 2014) studied the $\mathrm{M} / \mathrm{M} / \mathrm{c}$ queue model with client desertion which could treat as the Erlang-A Model which was sovereign and identically dispersed client discard period with an exponential distribution. Five statistical software packages for queuing theory compared by Chinthanie Fernando Ramasundarah ettige (Mary and Jayapriya, 2014).

\section{MATERIALS AND METHODS}

Queuing system: Queuing theory is the subject of arithmetic and its connected regions like the connected science and the subject of insights. It is infinitely useful in anticipating, distinguishing and assessing the

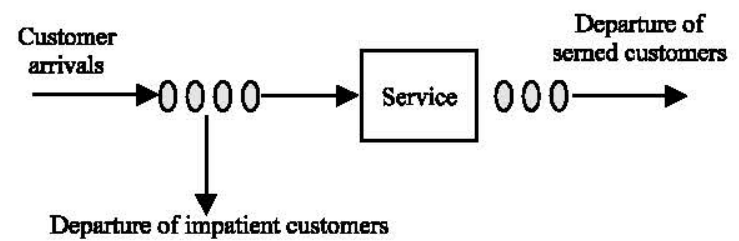

Fig. 1: Queuing model

execution of the framework. Standard lining hypothesis issues that were being seen by a few clients and the scientists are the clients going and going by a store, comparing to necessities approaching at a gadget. Lining hypothesis gives broadened time of general esteems. It is not conceivable or being considered in the lining framework system to distinguish or watch the event whether the following occasion will happen or not happen. A lining framework Fig. 1 can be said as "the clients set out to show up for a predefined check, pause if the check cannot start immediately and leave following being offered" the expression "client" can be men, items, machines and so on.

Arrivals may start as of single or a few sources alluded to as the populace. The populace that had called may be either restricted or 'boundless'. The landing procedure of the framework involves clarifying how the customers or the clients swing up to the framework which was signified by $\lambda$ (between entry rate). The administration instrument of a lining framework is exact regarding the number of servers (signified by $\mathrm{C}$ ) in which every server involves it has a line or a common line.

- M: Markovian (i.e., exponential)

- G: General distribution

- D: Deterministic

- Er: Erlang distribution

Queue capacity $(\mathrm{K})$ also, denotes the loss of customers if the queue is full. The size of the Population (P) can be either finite or infinite. Service discipline ( $\mathrm{Z}$ ) basically can take the following values:

- FCFS: First Come First Served

- LCFS: Last Come First Served

- Random: Service in Random order

- GD: General Discipline

The discipline of a queuing system explains in detail the process of whether a system follows a rule or a 
Table 1: System parameters and performance measures

\begin{tabular}{lll}
\hline Description & Symbols & Chosen input \\
\hline Inter arrivel rate & $\lambda$ & $0-2$ \\
Service rate & $\mu$ & 1,2 \\
Traffic intensity & $\rho$ & $0-1$ \\
Number of servers & $\mathrm{c}$ & 1,2 \\
Maximum numbers of service time & $\mathrm{K}$ & 4 \\
Capacity of waiting room & $\mathrm{r}$ & 4 \\
Coefficient of variation of service time & $\mathrm{C}$ & 1.5 \\
Erlang parameter & $\mathrm{er}$ & 2 \\
Mean number of customers in a system & $\mathrm{L}_{\mathrm{S}}$ & - \\
Mean number of customers in a queue & $\mathrm{W}_{\mathrm{S}}$ & - \\
Mean waiting time of customers in queue & $\mathrm{W}_{\mathrm{O}}$ & - \\
\hline
\end{tabular}

Table 2: Formulations-single server models

\begin{tabular}{lllll} 
Queue Model & $\mathrm{L}_{\mathrm{S}}$ & $\mathrm{L}_{0}$ & $\mathrm{~W}_{\mathrm{S}}$ & $\mathrm{W}_{0}$ \\
\hline $\mathrm{M} / \mathrm{M} / \mathrm{I}$ & $\rho / 1-\rho$ & $\rho^{2} / 1-\rho$ & $1 / \mu(1-\rho)$ & $\rho / \mu(1-\rho)$ \\
M/G/I & $\rho+\mathrm{A} \rho^{2} / 1-\rho$ & $\mathrm{A} \rho^{2 / 1-\rho}$ & $1 / \mu+\mathrm{A} \rho / \mu(1-\rho)$ & $\mathrm{A} \rho / \mu(1-\rho)$ \\
M/D/I & $\rho+\rho^{2} / 2(1-\rho)$ & $\rho^{2} / 2(1-\rho)$ & $2-\rho / 2 \mu(1-\rho)$ & $\rho / 2 \mu(1-\rho)$ \\
M/Er/I & $\rho+(1+1 / \mathrm{er}) \rho^{2} / 2(1-\rho)$ & $(1+1 / \mathrm{er}) \rho^{2} / 2(1-\rho)$ & $1 / \mu+(1+1 / \mathrm{er}) \rho / 2 \mu(1-\rho)$ & $(1+1 / \mathrm{er}) \rho / 2 \mu(1-\rho)$ \\
\hline
\end{tabular}

Table 3: Formulations for multi server models

\begin{tabular}{|c|c|}
\hline Values & Variables \\
\hline$P_{0}=\left[\sum_{n=0}^{c-1} \frac{\rho^{n}}{n !}+\frac{\rho^{c}}{c !(1-a)}\right]^{-1}$ & $\mathrm{c} !(1-\mathrm{a}) \mathrm{P}[\mathrm{N} \geq \mathrm{c}] \rho \mathrm{c}$ \\
\hline $\begin{array}{l}\mathrm{L}_{\mathrm{S}}=\mathrm{L}_{\mathrm{O}}+\rho \\
\mathrm{W}_{\mathrm{S}}=\mathrm{W}_{\mathrm{Q}}+1 / \mu\end{array}$ & $\begin{array}{l}\mathrm{L}_{0}=\rho P[N>c] / c(1-a) \\
\mathrm{W}_{0}=\mathrm{P}[\mathrm{N} \geq \mathrm{c}] / \mu \mathrm{c}(1-\mathrm{a})\end{array}$ \\
\hline$P_{n}=\frac{\rho^{n}}{n !} P_{0}$ & If $n \leq \mathrm{c}$ \\
\hline$P_{n}=\frac{\rho^{n}}{c ! c^{n-c}} P_{0}$ & If $n \geq c$ \\
\hline$P_{n}[N \geq n]=P_{0}\left[\sum_{k=n}^{c-1} \frac{\rho^{k}}{k !}+\frac{\rho^{c}}{c !(1-a)}\right.$ & If $\mathrm{n}<\mathrm{c}$ \\
\hline $\operatorname{Pn}[N \geq n]=P_{0}\left[a^{c} a^{n-c} / c !(1-a)\right]$ & if $n \geq c$ \\
\hline
\end{tabular}

regulation to a server that how the server identifies. It also, shows how the system selects the next customer or the next item from the existing queue or the queue under process from which the server completes the task given by a customer or a user.

Queuing model designs: The various set of parameters and various set of values assigned to the variables in the considered queuing model discussed in the following sections. The values assigned in the current tables are very much useful and helpful for the users in predicting or identifying the performance of the service systems whenever there is a chance of the existence of uncertainty in arrivals and service times to the system. The simplest possible queuing systems have the following components: customers, servers and a queue. An arriving customer is placed in the queue until a server is available. For the current work, it is assumed that clients had offered the services in which order they arrive in the system (First-Come-First-Served or FCFS).
Classification for the various set of system parameters and the chosen input values and performance measures for the queuing models considered had highlighted at Table 1 and 2 throughout it is assumed that the system is in steady state. The multi-server models and their parameter with their considered values shown in Table 3.

\section{RESULTS AND DISCUSSION}

The performance of the current cloud model with single and multi server's models ( $\mathrm{M} / \mathrm{G} / 1, \mathrm{M} / \mathrm{Er} / 1, \mathrm{M} / \mathrm{G} / \mathrm{c})$ conducted and the results were analysed for a various set of performance metrics. The assessment of performance metrics was carried out by programming in MATLAB ${ }^{\circledast}$ 7.60 environment developed by mathworks, Inc., USA. The input to the program is according to Table 1 and the output results for performance parameters for both single server models and multi-server models given at Fig. 2-5.

Performance evaluation for single servers indicate that as the service rate $(\mu)$ increases for a constant range of traffic intensity $(\rho)$ only waiting times of customers in the system $\left(\mathrm{W}_{\mathrm{S}}\right)$ and queue $\left(\mathrm{W}_{\mathrm{Q}}\right)$ decreases whereas the length of customers in system $\left(\mathrm{L}_{\mathrm{S}}\right)$ and queue $\left(\mathrm{L}_{\mathrm{Q}}\right)$ remain unchanged as it is independent on $\mu$. For the same input parameters, M/G/1 Model shows optimum performance regarding queue lengths and waiting times followed by $\mathrm{M} / \mathrm{Er} / 1$. Performance of $\mathrm{M} / \mathrm{G} / 1, \mathrm{M} / \mathrm{G} / \mathrm{c}$ shows detrimental nature when compared with other queuing models which had attributed to the higher value of $\mathrm{CoV}$. 

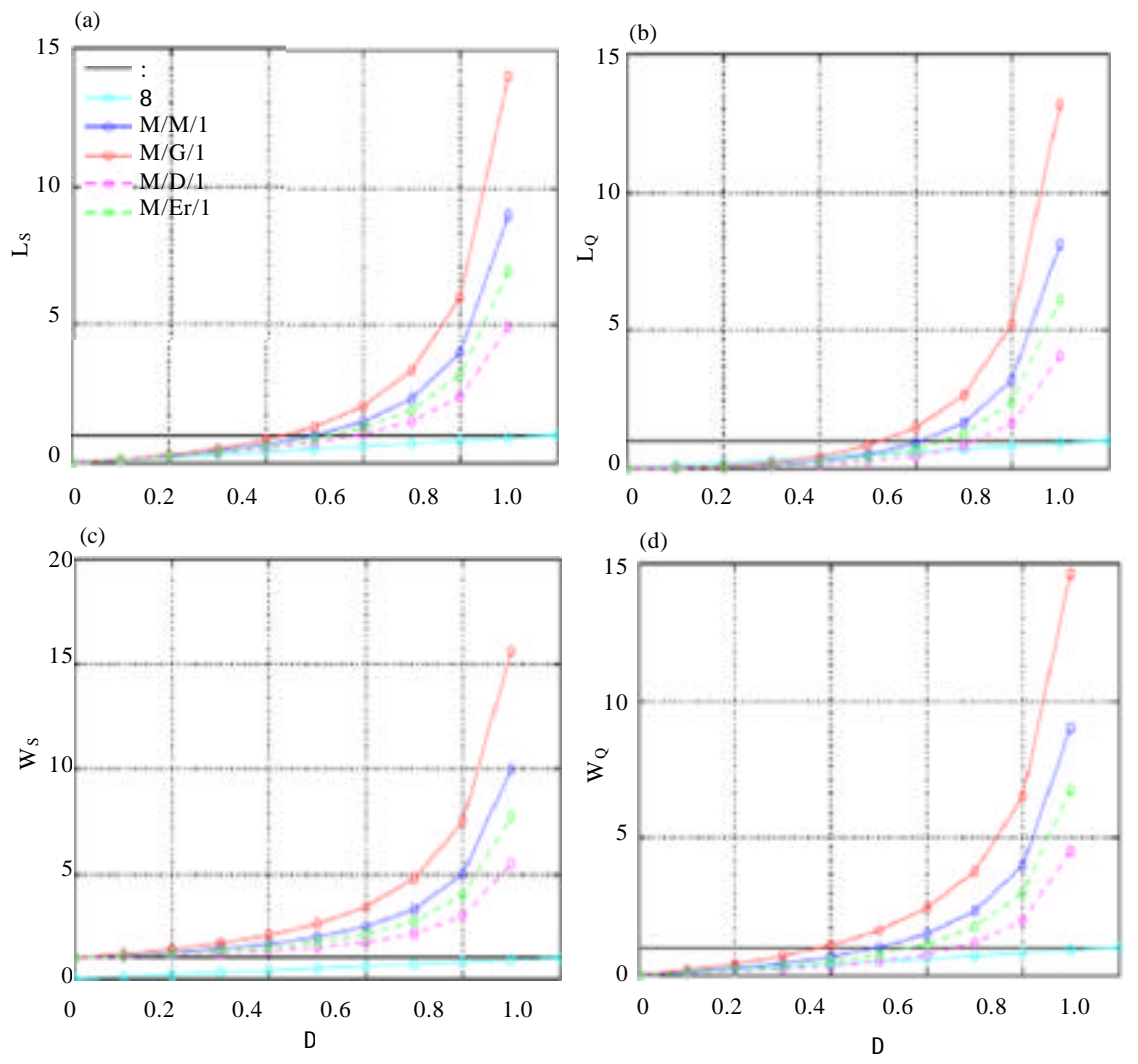

Fig. 2: Single server performance parameters, service rate $\mu=4$
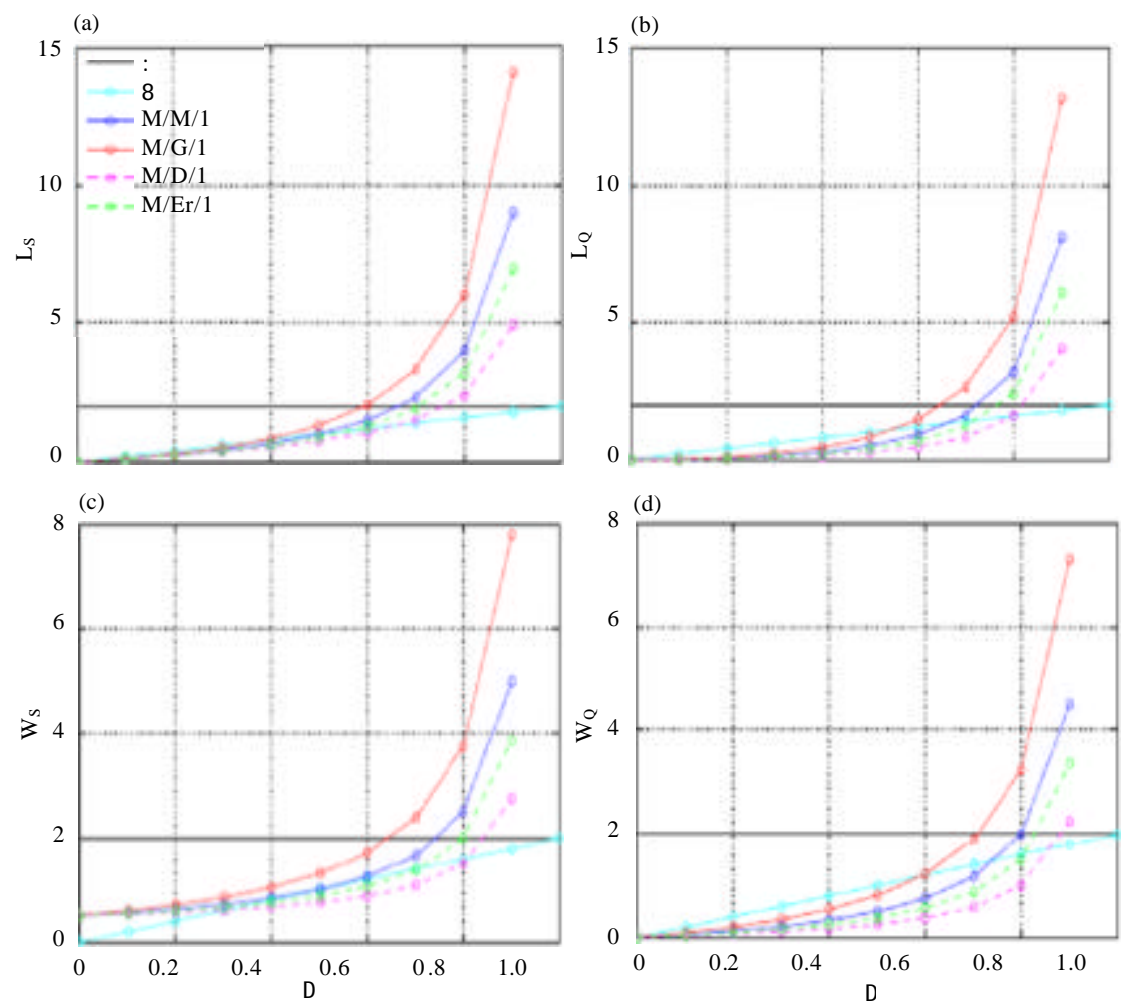

Fig. 3: Single server performance parameters, service rate $\mu=8$ 

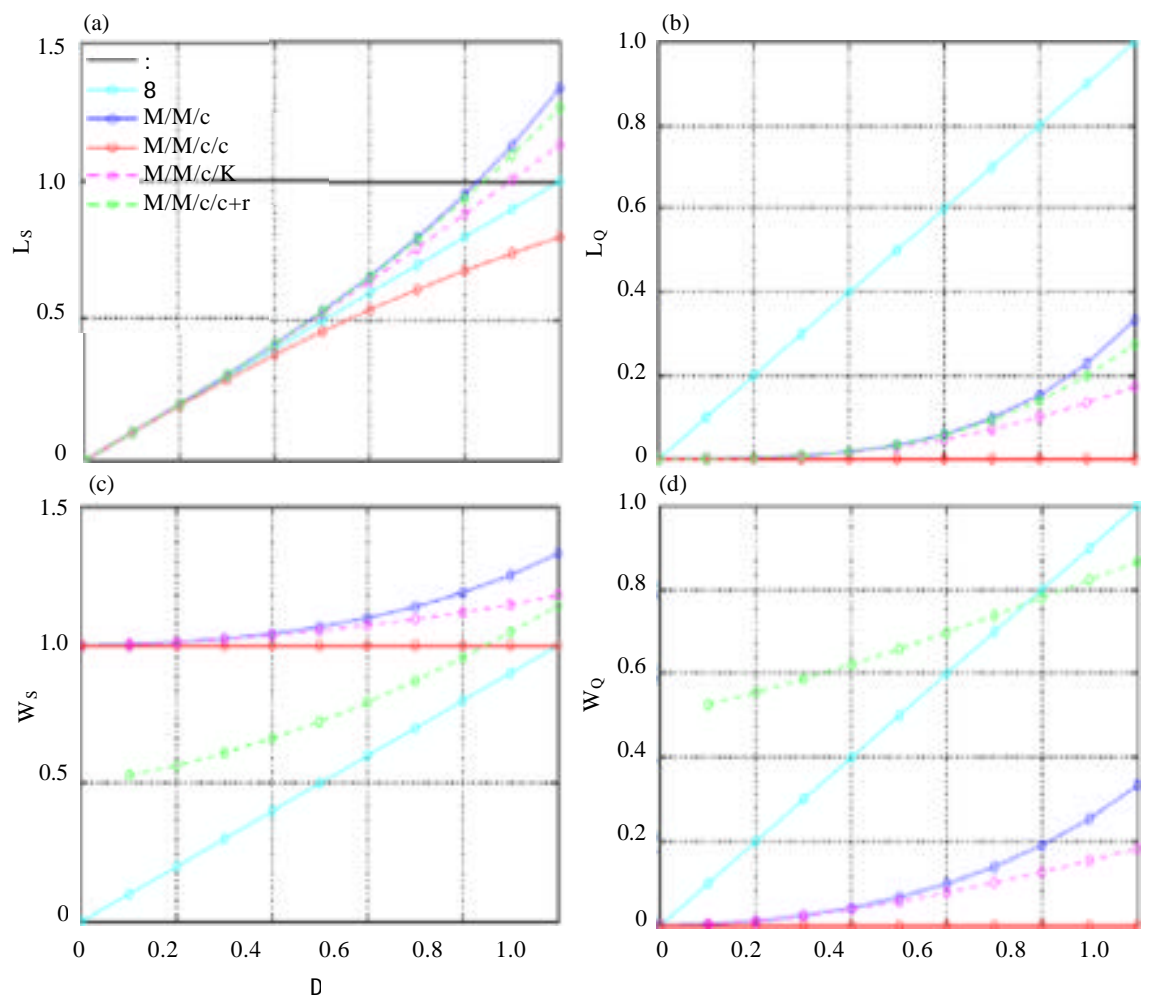

Fig. 4: Multi server performance parameters, service rate $\mu=4$
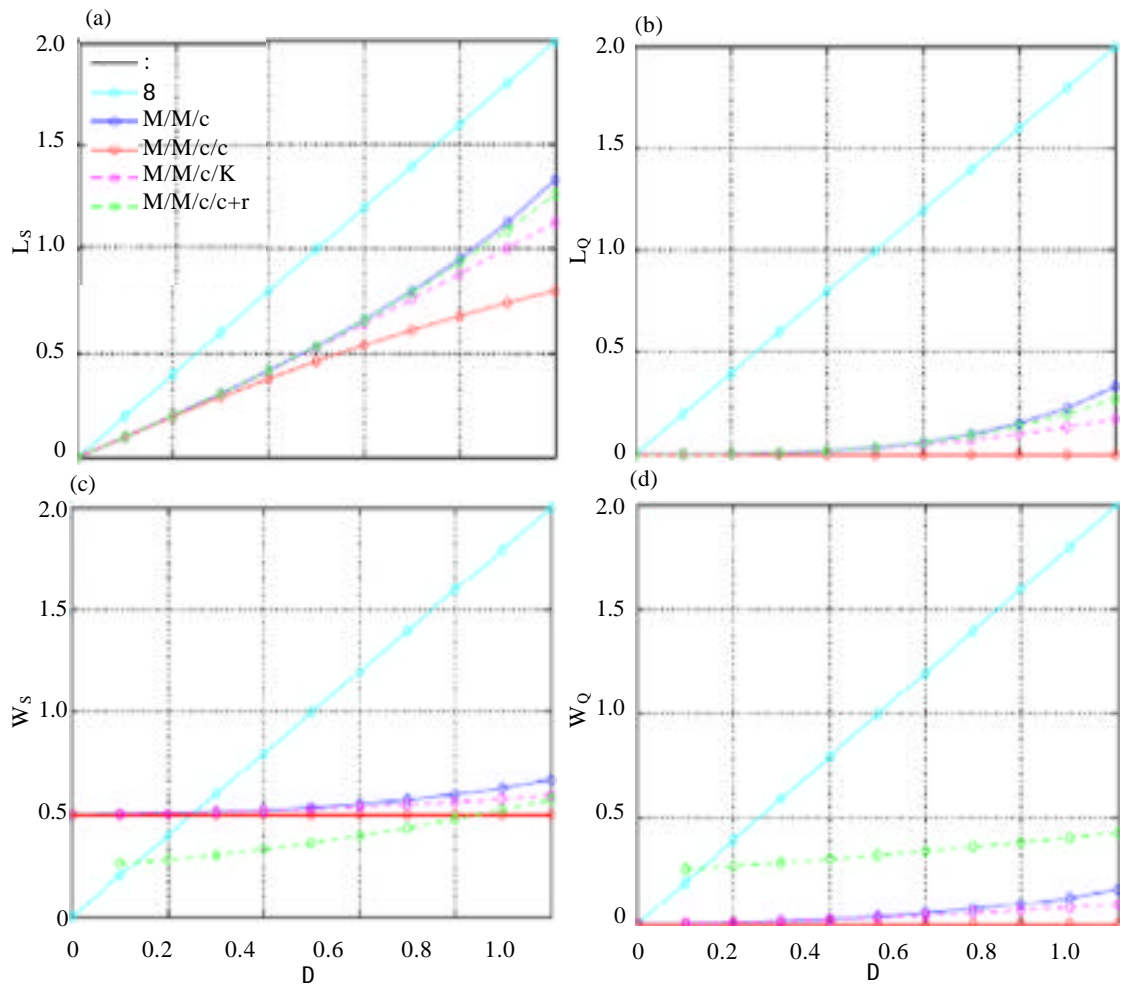

Fig. 5: Multi-server performance parameters, service rate $\mu=8$ 


\section{CONCLUSION}

Single server and numerous server models introduced alongside their definitions for execution parameters were analyzed. MATLAB programming/code and usage for execution assessment of distributed computing information server had performed and good in performance. Various models considered for the verification or the analysis of the behaviour of the cloud computing models with assumptions like the arrivals of data in bulk or in single had analysed. The results displayed in the form of some graphical representations. The results give us the impact that the arrival of packets of considerable data in the form of either in single packets or bulk packets will play a significant role in the performance of the cloud computing based model which was intended to be developed or implemented in the future cloud-based models with queuing system formats.

\section{REFERENCES}

Brandwajn, A. and H. Wang, 2008. A conditional probability approach to $\mathrm{M} / \mathrm{G} / 1$-like queues. Perform. Eval., 65: 366-381.
Hlynka, M. and S. Molinaro, 2010. Comparing expected wait times of a $\mathrm{M} / \mathrm{M} / 1$ queue. Master Thesis, Department of Mathematics and Statistics, University of Windsor, Windsor, Canada.

Khanghahi, N. and R. Ravanmehr, 2013. Cloud computing performance evaluation: Issues and challenges. Intl. J. Cloud Comput. Serv. Archit., 3: 29-41.

Mary, N.A.B. and K. Jayapriya, 2014. An extensive survey on QoS in cloud computing. Intl. J. Comput. Sci. Inf. Technol., 5: 1-5.

Mary, N.A.B. and K. Saravanan, 2013. Performance factors of cloud computing data centers using [(M/G/1): (8/Gdmodel)] queuing systems. Intl. J. Grid Comput. Appl., 4: 1-9.

Mathew, T.V., 2014. Queuing analysis, transportation systems engineering. Indian Institute of Technology Bombay, Mumbai, India.

Sowjanya, T.S., D. Praveen, K. Satish and A. Rahiman, 2011. The Queueing theory in cloud computing to reduce the waiting time. Intl. J. Comput. Sci. Eng. Technol., 1: 110-112.

Sztrik, J., 2012. Basic Queuing Theory. University of Debrecen, Debrecen, Hungary,.

Tripathi, A., 2013. Simulation of queuing models. Intl. J. Eng. Sci. Innovative Technol., 2: 195-199. 